\title{
The epidemiology of nontuberculous mycobacterial pulmonary disease in the Netherlands
}

\author{
Jodie Anne Schildkraut ${ }^{1}$, Sanne Maria Henriëtte Zweijpfenning ${ }^{2}$, Martijn $\mathrm{Nap}^{3}$, Kun $\mathrm{He}^{3}$, Elena Dacheva ${ }^{3}$, \\ Jetty Overbeek ${ }^{4}$, Alma Tostmann ${ }^{1}$, Heiman F.L. Wertheim ${ }^{1}$, Wouter Hoefsloot $\mathbb{D}^{2}$ and Jakko van Ingen $\mathbb{D}^{1}$
}

${ }^{1}$ Dept of Medical Microbiology, Radboudumc Center for Infectious Diseases, Radboud University Medical Center, Nijmegen, the Netherlands. ${ }^{2}$ Dept of Pulmonary Diseases, Radboudumc Center for Infectious Diseases, Radboud University Medical Center, Nijmegen, the Netherlands. ${ }^{3}$ IQVIA Analytics Solutions, Amsterdam, the Netherlands. ${ }^{4}$ PHARMO Institute for Drug Outcomes Research, Utrecht, the Netherlands.

Corresponding author: Jodie A. Schildkraut (jodie.schildkraut@radboudumc.nl)

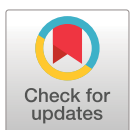

Copyright @The authors 202

This version is distributed under the terms of the Creative Commons Attribution NonCommercial Licence 4.0. For commercial reproduction rights and permissions contact permissions@ersnet.org

This article has supplementary material available from openres.ersjournals.com

Received: 23 March 2021 Accepted: 10 May 2021

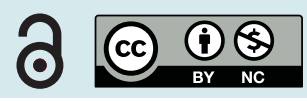

Shareable abstract (@ERSpublications)

The estimated annual prevalence of NTM-PD in the Netherlands using databases is between 2.3 and 5.9 patients per 100000 inhabitants. However, due to limitations within one database, an annual prevalence of between 2.3 and 4.5 is deemed more probable. https://bit.ly/3tOeTHG

Cite this article as: Schildkraut JA, Zweijpfenning SMH, Nap M, et al. The epidemiology of nontuberculous mycobacterial pulmonary disease in the Netherlands. ERJ Open Res 2021; 7: 002072021 [DOI: 10.1183/23120541.00207-2021].

\section{Abstract}

Background Nontuberculous mycobacteria (NTM) are emerging opportunistic pathogens of humans. Because NTM pulmonary disease (PD) is not a notifiable disease in Europe, the epidemiology of NTMPD is not well known. However, the prevalence of NTM-PD is thought to be increasing, particularly in countries where tuberculosis rates have decreased. Here we aim to determine the prevalence of NTM-PD in the Netherlands.

Methods Annual prevalence estimates of NTM-PD in the Netherlands (2012-2019) were derived from four separate databases, including two drug dispensing databases, an ICD-10 code database and a hospitalisation database. Databases covered a fraction of the Dutch population and were extrapolated. In addition, annual NTM-PD prevalence was also estimated by means of a pulmonologist survey.

Results The estimated annual prevalence of NTM-PD using databases is between 2.3 and 5.9 patients per 100000 inhabitants. Prevalence estimates derived from the drug dispensing databases, the hospitalisation database and the claims database were 2.3, 5.9, 3.5 and 4.5 per 100000 inhabitants, respectively. The annual prevalence estimated in the pulmonologist survey was between 6.2 and 9.9 per 100000 inhabitants. The annual prevalence remained stable over the included period.

Conclusion The estimated annual prevalence of NTM-PD using databases was between 2.3 and 5.9 patients per 100000 inhabitants. Due to the possible presence of tuberculosis patients and low coverage in one dispensing database, we believe an annual prevalence of between 2.3 and 4.5 patients per 100000 inhabitants is more probable, which still renders NTM-PD a serious health threat. This estimate is lower than the estimate from the pulmonologist survey, indicating physicians likely overestimate prevalence.

\section{Introduction}

Nontuberculous mycobacteria (NTM) are opportunistic pathogens of humans. The most frequent manifestation of NTM disease is a chronic pulmonary infection. Depending on host factors, this NTM pulmonary disease (NTM-PD) can be a severe fibrocavitary disease that resembles pulmonary tuberculosis or can be a milder nodular-bronchiectatic disease. NTM-PD is notorious for its highly complex, toxic and poorly efficacious treatments, where 1.5-2-year courses of three to four antibiotics yield cure rates of $50-60 \%$ at best [1].

As NTM-PD is not a notifiable disease anywhere in Europe, the epidemiology of NTM-PD in the region is not well known. Studies in Denmark (2000-2010), Scotland (1997-2008), Germany (2014) and the UK (2006-2016) have produced estimated annual prevalence rates of 2.43, 2.44, 3.3 and 6.4 cases per 100000 
per year, respectively [2-5]. Another study aimed at determining prevalence in five European countries estimated the prevalence to be 6.2 per 100000 inhabitants in 2016 [6]. Like in many countries where rates of tuberculosis infection have decreased, the prevalence of NTM-PD in the Netherlands is thought to be increasing [7]. However, the exact prevalence of NTM-PD in the Netherlands is unknown and clinical awareness remains low, and therefore, patients with suspected NTM-PD may not be subsequently diagnosed and recognised.

By using existing data sources on drug prescriptions and disease registration codes, we wanted to make an estimate of the prevalence of NTM-PD in the Netherlands, to determine the scale of the problem and raise clinical awareness, thereby contributing to improved recognition and diagnosis.

Methods

To come to a reliable estimation of the prevalence of NTM-PD in the Netherlands over the time period 2012-2019, we have collated data from pharmacy drug dispensing databases, primary care diagnostic code databases, hospitalisation databases and from a survey among pulmonologists.

\section{Drug prescription databases}

We accessed two independent drug prescription databases. The first was IQVIA's Real-World Data Longitudinal Prescription database (LRx, Amsterdam, the Netherlands) [8]. The LRx database contains anonymised prescription records from hospital and community pharmacies. Data are included regarding patient characteristics, all dispensed medication (including dose), dispensing pharmacy, date and length of prescription. Overall, approximately 57\% of outpatient prescriptions for NTM infections (based specifically on the five NTM drugs in focus, see table 1) within the Netherlands are included in the LRx database, although coverage was difficult to determine as it differed per included antibiotic in this study. Secondly, the Outpatient Pharmacy Database of the PHARMO Database Network (PHARMO) was accessed, with a coverage of approximately $28 \%$ for outpatient dispensing per year [9].

These drug prescription databases were used to define both "probable NTM-PD patients" (three-drug regimens) and "possible NTM-PD patients" (two-drug regimens) based on drug combinations used for NTM-PD treatment, shown in table 1. Both databases were searched for prescriptions of the drug combinations presented in table 1, in the 2015-2019 (IQVIA) or 2013-2017 (PHARMO) periods; only patients receiving treatment for $>30$ days were included, as treatment for $<30$ days was deemed too short for NTM-PD. The IQVIA database was additionally queried for prescriptions of regimens for the most prevalent causative agents of NTM-PD (Mycobacterium avium complex, Mycobacterium malmoense and Mycobacterium kansasii) [10]. These regimens included at least two of the following molecules: rifampicin, rifabutin, ethambutol, clarithromycin and azithromycin. Unfortunately, these combinations do not cover rapidly growing NTM. Prescriptions of clindamycin were used to enable exclusion of patients treated for prosthetic joint infections [11]. All patients with regimens that at one point contained pyrazinamide for $>30$ days were excluded, assuming that these were tuberculosis patients. All data were anonymised according to Dutch and European privacy legislation through a trusted third party.

\section{TABLE 1 Nontuberculous mycobacteria pulmonary disease (NTM-PD) treatment definitions}

\begin{tabular}{lc} 
Definition of likelihood of NTM-PD treatment & Drug combination \\
\hline Probable & Rifampicin-ethambutol-azithromycin \\
\hline Probable & Rifampicin-ethambutol-clarithromycin \\
\hline Probable & Rifabutin-ethambutol-azithromycin \\
\hline Probable & Rifabutin-ethambutol-clarithromycin \\
\hline Probable & Clofazimine-ethambutol-azithromycin \\
\hline Probable & Clofazimine-ethambutol-clarithromycin \\
Possible & Rifampicin-ethambutol \\
Possible & Rifampicin-azithromycin \\
\hline Possible & Rifabutin-clarithromycin \\
Possible & Rifabutin-ethambutol \\
\hline Possible & Rifabutin-azithromycin \\
\hline Possible & Rifabutin-clarithromycin \\
Possible & Ethambutol-azithromycin \\
Possible & Ethambutol-clarithromycin \\
\hline
\end{tabular}


To estimate the overall annual prevalence of NTM-PD infections in the Netherlands, the total number of probable and possible NTM-PD patients was determined using the mean of the Dutch population census (https://opendata.cbs.nl/statline/\#/CBS/nl/dataset/37556/table?ts=1581944556208) in 2013-2017 (PHARMO, 17004781) or 2015-2019 (IQVIA, 17084920) as denominators. Prevalence estimates were then corrected for the coverage of the databases, the proportion of NTM-PD patients that remain untreated, estimated from two previous studies (48\%), and inability of these drugs to identify the proportion of NTM-PD caused by Mycobacterium abscessus and other rapidly growing NTM (20\%) [12-14]; an overview of all extrapolations are shown in supplemental table S1.

\section{Diagnostic codes database}

In the Netherlands, health insurance claims are submitted by the treating physician and claimed from the insurance companies by the hospital based on the official diagnosis code (International Statistical Classification of Diseases and Related Health Problems (ICD)-10), descriptions of which can be found online (www.whofic.nl/familie-van-internationale-classificaties/referentie-classificaties/icd-10). IQVIA's claims database is comprised of the claims submitted to a subset of these health insurance companies, representing about a quarter of the Dutch population. The healthcare insurance claims data contained an anonymous patient ID, claim indication, specialist involved, official diagnosis code (ICD-10) and diagnosis description. In our study we selected all patients with ICD-10 code A31.0 (NTM-PD code) in the 2012-2016 period, referred to as "confirmed NTM-PD patients". These data were used to estimate an annual prevalence of confirmed NTM-PD, using the mean population census from 2012-2016 as a denominator [12]. Prevalence estimates were then corrected for the limited sensitivity (50\%) of ICD-10 codes for NTM-PD seen in previous studies [15].

\section{Hospitalisation database}

In addition to the previous methods, we calculated the annual prevalence of patients with a hospital admission for NTM-PD (ICD-10 code A31.0) in the period from 2014 to 2017 using the Hospitalization Database of the PHARMO Database Network, extended with data from the Radboudumc Center of Expertise for mycobacterial disease, only patients with a hospital admission requiring a hospital stay were included. The overlap between the catchment area of the Hospitalization Database and the catchment area of the NTM Center of Expertise could not be determined. Therefore, we calculated a conservative and liberal estimate of annual NTM-PD prevalence of patients with a hospital admission for NTM-PD by using either the number of people in the catchment area of hospitals in the PHARMO Database Network (liberal) or total population in the Netherlands (conservative) as denominators for the calculations. Findings were then extrapolated to total NTM-PD prevalence using estimates of NTM-PD hospitalisation rate from Germany, approximately 14.7\%, based on an annual average of 0.91 hospitalisations per 100000 population between 2005-2011 and an overall prevalence of NTM-PD of 6.2 per 100000 inhabitants in $2016[6,16]$.

\section{Pulmonologist survey}

To assess the clinical perception of the prevalence of NTM-PD, we performed a survey among pulmonologists in the Netherlands in 2018. The survey was completed via a secure online platform and 154 participants were approached; no reminder was sent. The survey addressed a broader topic of diagnostic testing for NTM in patients with bronchiectasis and was aimed at hospital-based pulmonologists who had consulted NTM-PD patients and at least 10 patients with bronchiectasis in the previous 12 months [17]. The respondents were asked to estimate the number of patients with NTM-PD they consulted in the previous 12 months. Based on the total number of registered pulmonologists, we estimated the prevalence of patients with NTM-PD. To estimate the NTM-PD prevalence based on expert opinion, we used the 2018 Dutch national population census and the number of registered pulmonologists, as recorded by the Netherlands Society for Pulmonary Diseases and Tuberculosis Physicians (www.nvalt.nl). A conservative estimation was calculated by excluding pulmonologists who had treated fewer than 10 bronchiectasis patients in the previous year assuming they also had not treated NTM-PD patients, and a liberal estimation was calculated by assuming all pulmonologist treated the same number of NTM patients as was indicated by the pulmonologists who were included in the questionnaire study.

\section{Data analysis}

Statistical analysis was performed using $\mathrm{R}$ version 3.3.3. The prevalence was determined as the total number of individuals in each database per year, divided by the Dutch population in each year (per 100000) using the Dutch national population census. A 95\% CI was calculated for each prevalence both before and after extrapolation. 
Results

Drug databases

Using the "probable" case definition for NTM-PD treatment, we identified a total of 174 patients with a combination of a rifamycin, ethambutol and a macrolide from PHARMO in the study period (2013-2017). From the IQVIA LRx data (2015-2019), we identified 356 patients with a prescription for the triple-drug regimen. Using the criteria for "possible NTM-PD" (at least two of the three drugs in the triple-drug regimen), the PHARMO and IQVIA database yielded 373 and 430 patients possibly treated for NTM-PD, respectively. The annual prevalence of NTM-PD that required antibiotic treatment using the "probable NTM-PD” and "possible NTM-PD” case definitions of treatment was 0.20 per 100000 (95\% CI 0.14-0.26) and 0.64 per 100000 (95\% CI 0.48-0.80), respectively, in the PHARMO database. Using data from the IQVIA LRx database the criteria for "probable NTM-PD” yielded an annual prevalence of 0.42 per 100000 (95\% CI 0.32-0.51) while the criteria for "probable NTM-PD” and “possible NTM-PD” yielded an annual prevalence of 0.50 per 100000 (95\% CI 0.39-0.62). After correction for database coverage, untreated patients and proportion of NTM-PD caused by M. abscessus, projected NTM-PD prevalence estimates for a combination of "possible NTM" and "probable NTM" patients were 5.9 per 100000 (95\% CI 4.9-7.0) and 2.3 per 100000 (95\% CI 1.9-2.7) in the PHARMO and IQVIA database, respectively (figure 1).

\section{Diagnostic codes database}

In 2015 and 2016, there were 470 patients with the ICD-10 code A31.0 for NTM-PD in IQVIA's claims database. Based on the Dutch population in 2015, this corresponds to an annual prevalence of 0.56 per 100000 (95\% CI 0.42-0.70). After correction for database coverage and limited sensitivity of ICD-10 codes this yields an annual projected NTM-PD prevalence of 4.5 per 100000 (95\% CI 3.3-5.6) (figure 1).

\section{Hospitalisation database}

Between 2014 and 2017 there are 150 patients in the Hospitalisation Database of the PHARMO Database Network and 86 patients in the Radboudumc Center of Expertise. This means that there were between 0.4 (95\% CI 0.3-0.4) and 0.51 (95\% CI 0.44-0.58) NTM-PD hospital admissions per 100000 population per year, using the conservative and liberal estimation methods. Annual prevalence estimates were then extrapolated by correcting for the estimated hospitalisation rate, yielding an annual projected NTM-PD prevalence of between 2.4 (95\% CI 1.8-3.0) and 3.48 (95\% CI 3.0-4.0) patients per 100000 inhabitants, shown in figure 1.

\section{Pulmonologist survey}

The survey was completed by 66 pulmonologists (42.9\% response rate) of which 64 indicated that they spent $>80 \%$ of their time in a clinical setting. Of the remaining 64 pulmonologists, 40 (62.5\%) had treated NTM-PD patients and $>10$ bronchiectasis patients over the past 12 months and were therefore eligible for inclusion in the analysis. In addition, of these 64 pulmonologists, $65 \%$ indicated that they had treated NTM-PD cases, with an estimated average of four NTM-PD patients per year. The conservative prevalence estimate yielded an annual prevalence of 6.2 per 100000 , while the liberal prevalence estimate was 9.9 per 100000 (figure 1).

\section{Discussion}

This is the first study on NTM epidemiology in the Netherlands using hospital, pharmacy and diagnostic codes databases as well as a physician survey; the extrapolated prevalence estimates of each database query

a)

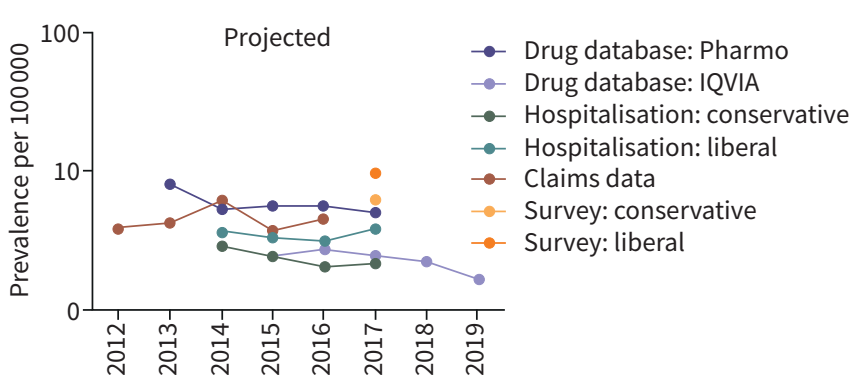

b)

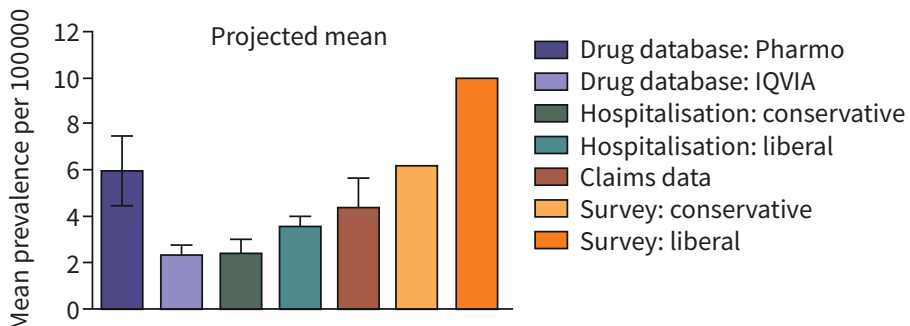

FIGURE 1 Projected prevalence. a) Annual projected prevalence by database per year. b) Mean projected annual prevalence by database. For drug database prevalence estimates the lenient definition of nontuberculous mycobacteria pulmonary disease (NTM-PD) was included. 
and the survey are shown in figure 1. Overall, we estimate the mean annual prevalence to be between 2.3 and 5.9 per 100000 inhabitants per year. The strength and limitations and the overall conclusion of each of the approaches are summarised in table 2.

Using the drug dispensing databases of both the PHARMO institute and IQVIA we recorded 0.64 (95\% CI 0.48-0.80) and 0.50 (95\% CI 0.39-0.62) patients per 100000 inhabitants per year receiving NTM-PD treatment applying the lenient definition thereof. However, both databases underestimate prevalence as they do not cover all pharmacies within the Netherlands and drug prescription databases only detect patients receiving treatment with the predefined antibiotics. To estimate the projected prevalence of NTM-PD in the Netherlands, we extrapolated our findings using database coverage data, inclusion of untreated NTM-PD patients and inclusion of M. abscessus patients. Based on the respective coverage percentages of both databases and acknowledging low compliance with guidelines [14], the number of patients receiving the "possible NTM-PD" and "probable NTM-PD" definitions of NTM-PD treatment was extrapolated. In addition, previous studies have shown that for a significant portion of NTM-PD patients the decision is made to not treat.

We corrected for untreated patients (48\%, average of findings in previous studies) and $M$. abscessus/rapid growers (20\% of NTM-PD caseload in most European Union (EU) countries) [10, 12-14] amounting to a total projected mean annual NTM-PD prevalence of 5.9 per 100000 (95\% CI 4.9-7.0) and 2.3 per 100000

\section{TABLE 2 Overview of data sources, outcomes, strengths and limitations}

\begin{tabular}{|c|c|c|c|c|c|}
\hline Data source & $\begin{array}{c}\text { Estimated } \\
\text { prevalence } \\
\text { per } 100000 \\
(95 \% \mathrm{Cl})\end{array}$ & $\begin{array}{c}\text { Range } \\
\text { conservative-liberal } \\
\text { per } 100000\end{array}$ & $\begin{array}{l}\text { Major strength } \\
\text { of this } \\
\text { approach }\end{array}$ & $\begin{array}{c}\text { Major limitation of this } \\
\text { approach }\end{array}$ & Overall conclusion \\
\hline $\begin{array}{l}\text { Pharmacy dispensing } \\
\text { data: antibiotics } \\
\text { specific for NTM-PD } \\
\text { treatment }\end{array}$ & $\begin{array}{c}\text { IQVIA } \\
2.3(1.9-2.7) \\
\text { PHARMO } \\
5.9(4.9-7.0)\end{array}$ & $\mathrm{N} / \mathrm{A}$ & $\begin{array}{l}\text { These } \\
\text { antibiotics are } \\
\text { specific for } \\
\text { NTM-PD, so the } \\
\text { data are } \\
\text { reliable }\end{array}$ & $\begin{array}{l}\text { Not all antibiotics that are } \\
\text { used for NTM-PD are } \\
\text { included } \\
\text { However, the indication of } \\
\text { the other antibiotics is not } \\
\text { specific enough to use in } \\
\text { this context }\end{array}$ & $\begin{array}{c}\text { Probably an } \\
\text { underestimation, because } \\
\text { for many NTM-PD patients } \\
\text { no treatment is initiated } \\
\text { and not all NTM-PD } \\
\text { antibiotics were included }\end{array}$ \\
\hline $\begin{array}{l}\text { Healthcare insurance } \\
\text { data: ICD-10 codes }\end{array}$ & $4.5(3.3-4.6)$ & $\mathrm{N} / \mathrm{A}$ & $\begin{array}{l}\text { ICD-10 codes } \\
\text { are specific for } \\
\text { NTM-PD and } \\
\text { therefore } \\
\text { reliable }\end{array}$ & $\begin{array}{c}\text { Previous studies have } \\
\text { shown that ICD-10 codes } \\
\text { have limited sensitivity } \\
\text { when detecting NTM-PD } \\
\text { cases as the ICD-10 code } \\
\text { for NTM-PD is often not } \\
\text { assigned }\end{array}$ & $\begin{array}{c}\text { Probably an } \\
\text { underestimation, because } \\
\text { many patients are not } \\
\text { assigned the ICD-10 code } \\
\text { for NTM-PD }\end{array}$ \\
\hline $\begin{array}{l}\text { Expert opinion: } \\
\text { pulmonologists } \\
\text { estimating number of } \\
\text { patients with } \\
\text { bronchiectasis and } \\
\text { NTM-PD under their } \\
\text { responsibility }\end{array}$ & 6.2 & $6.2-9.9$ & & $\begin{array}{l}\text { Very subjective, can be } \\
\text { biased by personal } \\
\text { experience of } \\
\text { pulmonologist } \\
\text { Lower estimate does not } \\
\text { account for NTM patients } \\
\text { treated by pulmonologists } \\
\text { that see }<10 \text { bronchiectasis } \\
\text { patients while upper } \\
\text { estimate was probably over } \\
\text { extrapolated as physicians } \\
\text { treating fewer } \\
\text { bronchiectasis patients } \\
\text { probably also treat fewer } \\
\text { NTM-PD patients }\end{array}$ & Overestimation \\
\hline
\end{tabular}


(95\% CI 1.9-2.7) for PHARMO and the IQVIA LRx databases, respectively. The relatively large difference in prevalence estimated from each drug database is likely due to two factors. First, within the IQVIA database, patients simultaneously receiving pyrazinamide treatment were excluded, as these are likely to be tuberculosis patients and not NTM-PD. Due to the absence of data on pyrazinamide use this was not possible in the PHARMO database. Therefore, the relatively small amount of tuberculosis patients receiving ethambutol treatment for $>30$ days likely remain within our selection, leading to an overestimation of NTM-PD patients. The second being the difference in coverage between the two databases. Overall, the IQVIA database has a much higher coverage, meaning extrapolation likely introduces less error. In addition, coverage of certain areas within the Netherland differs between each database, possibly influencing prevalence estimates, and although the total period included for each database is equal (5 years), the specific years included differs meaning estimates can be influenced by annual fluctuations in prevalence.

Based on the diagnostic codes database the annual prevalence of the ICD-10 code A31.0 for NTM-PD after correction for coverage and low sensitivity of ICD-10 codes was at 4.5 per 100000 (95\% CI 3.3-5.6) [15]. While extrapolation of Dutch hospitalisation rates to total NTM-PD prevalence amounted to an annual prevalence between 2.4 (95\% CI 1.8-3.0) and 3.5 (95\% CI 3.0-4.0) patients per 100000 inhabitants. However, due to the possible presence of tuberculosis patients and relatively low coverage of dispensing pharmacies in the Netherlands, the PHARMO institute dispensing database estimate is probably less reliable than the others. Therefore, the lower estimates of mean annual prevalence, between 2.3 and 4.5 per 100000 , are deemed more probable.

There are important limitations inherent to assessments of disease prevalence from prescription, disease code and hospitalisation databases and physician estimates. Our criteria to query the drug dispensing databases were strict, given the well-known poor compliance (10\% in five EU countries) with NTM-PD treatment guidelines [14]. The projections, too, are biased as they were based on studies from other countries and health systems [6,12, 13, 15-17]. Nonetheless, we do provide a first assessment of NTM-PD annual prevalence in the Netherlands, which is between 2.3 and 5.9 patients per 100000 inhabitants in the study period. This is in line with previous studies in neighbouring Germany and the UK [3-6]. We did not see an increase during the studied period. This finding is in line with data from the national database in Denmark that also showed a stable prevalence of NTM-PD over a 25-year period, but in contrast with multiple studies that claim NTM-PD prevalence is increasing $[1,3-5,16,18]$. National surveillance of NTM-PD would be valuable to better characterise disease burden over time. Laboratory-based surveillance could be a first step and has been successfully implemented before [19]. A previous laboratory-based prevalence estimate of NTM-PD in the Netherlands found an annual prevalence of 5.5 patients per 100000 inhabitants [6]. This finding falls within the 2.3 and 5.9 patients per 100000 prevalence estimate, but is slightly higher than the more probable estimate of between 2.3 and 4.5 per 100000 .

In conclusion, after extrapolation of data retrieved from each database the estimated annual prevalence of NTM-PD using databases is between 2.3 and 5.9 patients per 100000 inhabitants, as shown in figure 1 . However, due to the possible presence of tuberculosis patients and relatively low coverage of dispensing pharmacies in one dispensing database, a prevalence of between 2.3 and 4.5 per 100000 is deemed more probable. This is lower than the annual prevalence estimated in the pulmonologist survey, which was between 6.2 and 9.9 per 100000 inhabitants annually, indicating that physicians likely overestimate prevalence. Our databases show that annual prevalence remains relatively stable over the period included in our survey. This disease prevalence does show that we need to increase efforts to understand this disease, its risk factors and to optimise its treatment.

Conflict of interest: J.A. Schildkraut has nothing to disclose. S.M.H. Zweijpfenning has nothing to disclose. M. Nap reports a grant from InsMed during the conduct of the study. K. He reports grants from InsMed during the conduct of the study. E. Dacheva reports grants from InsMed during the conduct of the study. J. Overbeek reports is an employee of the PHARMO Institute for Drug Outcomes Research. This independent research institute performs financially supported studies for government and related healthcare authorities and several pharmaceutical companies. A. Tostmann has nothing to disclose. H.F.L. Wertheim has nothing to disclose. W. Hoefsloot has nothing to disclose. J. van Ingen reports advisory board membership for InsMed outside the submitted work.

References

1 Cowman S, van Ingen J, Griffith DE, et al. Non-tuberculous mycobacterial pulmonary disease. Eur Respir J 2019; 54: 1900250. 
2 Andréjak C, Thomsen V, Johansen IS, et al. Nontuberculous pulmonary mycobacteriosis in Denmark: incidence and prognostic factors. Am J Respir Crit Care Med 2010; 181: 514-521.

3 Russell CD, Claxton P, Doig C, et al. Non-tuberculous mycobacteria: a retrospective review of Scottish isolates from 2000 to 2010. Thorax 2014; 69: 593-595.

4 Ringshausen FC, Wagner D, de Roux A, et al. Prevalence of nontuberculous mycobacterial pulmonary disease, Germany, 2009-2014. Emerg Infect Dis 2016; 22: 1102-1105.

5 Axson EL, Bloom Cl, Quint JK. Nontuberculous mycobacterial disease managed within UK primary care, 2006-2016. Eur J Clin Microbiol Infect Dis 2018; 37: 1795-1803.

6 Schildkraut JA, Gallagher J, Morimoto K, et al. Epidemiology of nontuberculous mycobacterial pulmonary disease in Europe and Japan by Delphi estimation. Respir Med 2020; 173: 106164.

7 van Ingen J, Bendien SA, de Lange WC, et al. Clinical relevance of non-tuberculous mycobacteria isolated in the Nijmegen-Arnhem region, the Netherlands. Thorax 2009; 64: 502-506.

8 IQVIA. IQVIA Longitudinal Prescription Data (LRx) Fact Sheet. www.iqvia.com/locations/united-kingdom/ library/fact-sheets/iqvia-longitudinal-prescription-data-Irx. Date last updated: July 21, 2020. Date last accessed: February 2, 2021.

9 Kuiper JG, Bakker M, Penning-van Beest FJA, et al. Existing data sources for clinical epidemiology: the PHARMO database network. Clin Epidemiol 2020; 12: 415-422.

10 Zweijpfenning S, Kops S, Magis-Escurra C, et al. Treatment and outcome of non-tuberculous mycobacterial pulmonary disease in a predominantly fibro-cavitary disease cohort. Respir Med 2017; 131: 220-224.

11 Leijtens B, Elbers JBW, Sturm PD, et al. Clindamycin-rifampin combination therapy for staphylococcal periprosthetic joint infections: a retrospective observational study. BMC Infect Dis 2017; 17: 321.

12 Diel R, Obradovic M, Tyler S, et al. Real-world treatment patterns in patients with nontuberculous mycobacterial lung disease in general and pneumologist practices in Germany. J Clin Tuberc Other Mycobact Dis 2020; 20: 100178.

13 Rawson TM, Abbara A, Kranzer K, et al. Factors which influence treatment initiation for pulmonary non-tuberculous mycobacterium infection in HIV negative patients; a multicentre observational study. Respir Med 2016; 120: 101-108.

14 van Ingen J, Wagner D, Gallagher J, et al. Poor adherence to management guidelines in nontuberculous mycobacterial pulmonary diseases. Eur Respir J 2017; 49: 1601855.

15 Winthrop KL, Baxter R, Liu L, et al. The reliability of diagnostic coding and laboratory data to identify tuberculosis and nontuberculous mycobacterial disease among rheumatoid arthritis patients using anti-tumor necrosis factor therapy. Pharmacoepidemiol Drug Saf 2011; 20: 229-235.

16 Ringshausen FC, Apel RM, Bange FC, et al. Burden and trends of hospitalisations associated with pulmonary non-tuberculous mycobacterial infections in Germany, 2005-2011. BMC Infect Dis 2013; 13: 231.

17 Wagner D, van Ingen J, van der Laan R, et al. Non-tuberculous mycobacterial lung disease in patients with bronchiectasis: perceived risk, severity and guideline adherence in a European physician survey. BMJ Open Respir Res 2020; 7.

18 Hermansen TS, Ravn P, Svensson E, et al. Nontuberculous mycobacteria in Denmark, incidence and clinical importance during the last quarter-century. Sci Rep 2017; 7: 6696.

19 Jankovic M, Sabol I, Zmak L, et al. Microbiological criteria in non-tuberculous mycobacteria pulmonary disease: a tool for diagnosis and epidemiology. Int J Tuberc Lung Dis 2016; 20: 934-940. 